\title{
Cervical Cord Atrophy and Long-Term Disease Progression in Patients with Primary-Progressive Multiple Sclerosis
}

\author{
(D)F.X. Aymerich, (DC. Auger, (D). Alonso, (D) M. Alberich, (D). Sastre-Garriga, (D) M. Tintoré, (D)X. Montalban, and (D) A. Rovira
}

\begin{abstract}
BACKGROUND AND PURPOSE: Cervical cord atrophy has been associated with clinical disability in multiple sclerosis and is proposed as an outcome measure of neurodegeneration. The aim of this study was to quantify the development of cervical cord atrophy and to evaluate its association with disability progression in patients with primary-progressive multiple sclerosis.
\end{abstract}

MATERIALS AND METHODS: Thirty-one patients with primary-progressive multiple sclerosis underwent $1.5 \mathrm{~T}$ brain and spinal cord MR imaging at baseline and 6-7 years later. The cervical spinal cord from $\mathrm{Cl}$ to $\mathrm{C5}$ was segmented to evaluate the normalized overall cross-sectional area and the cross-sectional area of C2-C3, C3-C4, and C4-C5. The annualized rates of normalized cross-sectional area loss were also evaluated. To estimate clinical progression, we determined the Expanded Disability Status Scale score at baseline and at 2 and 14 years after baseline to compute the normalized area under the curve of the Expanded Disability Status Scale and the Expanded Disability Status Scale changes from baseline to the follow-up time points. Associations between the cord cross-sectional area and brain MR imaging and clinical measures were also investigated. Finally, the value of all these measures for predicting long-term disability was evaluated.

RESULTS: Some normalized cross-sectional area measurements showed moderate correlations with the normalized area under the curve of the Expanded Disability Status Scale, ranging from -0.439 to $-0.359(P<.05)$. Moreover, the annualized rate of the normalized mean cross-sectional area loss and the baseline Expanded Disability Status Scale were independent predictors of long-term disability progression.

CONCLUSIONS: These data indicate that development of cervical cord atrophy is associated with progression of disability and is predictive of this event in patients with primary-progressive MS.

ABBREVIATIONS: aNMCSA = annualized normalized mean cross-sectional area loss rate between the baseline and follow-up examination; $B P F=$ brain parenchymal fraction; CSA = cross-sectional area; EDSS = Expanded Disability Status Scale; NMCSA = normalized mean CSA; NCSA23 = normalized CSA at the C2-C3 level; NCSA34 = normalized CSA at the C3-C4 level; NCSA45 = normalized CSA at the C4-C5 level; PPMS = primary-progressive multiple sclerosis; TILV = TI lesion volume; $\mathrm{T} 2 \mathrm{LV}=\mathrm{T} 2$ lesion volume

$\mathbf{P}$ rimary-progressive multiple sclerosis (PPMS) is characterized by sustained progression of disability from disease onset and is typically associated with severe motor impairment. ${ }^{1,2}$ The rate at which disability progresses is highly variable, but impairment

Received August 8, 2017; accepted after revision October 16.

From the Magnetic Resonance Unit (F.X.A., C.A., J.A., M.A., A.R.), Department of Radiology, and Centre d'Esclerosi Múltiple de Catalunya (J.S.-G., M.T., X.M.), Department of Neurology/Neuroimmunology, Hospital Universitari Vall d'Hebron, Universitat Autònoma de Barcelona, Barcelona, Spain; and Department of Automatic Control (F.X.A.), Universitat Politècnica de Catalunya-Barcelona Tech, Barcelona, Spain.

Paper previously presented, in part, at: Annual Meeting of the European Committee for Treatment and Research in Multiple Sclerosis, October 7-10, 2015; Barcelona, Spain.

Please address correspondence to F. Xavier Aymerich, PhD, Magnetic Resonance Unit, Hospital Universitari Vall d'Hebron, P. Vall d'Hebron 119-129, 08035 Barcelona, Spain; e-mail: xavier.aymerich@idi.gencat.cat

http://dx.doi.org/10.3174/ajnr.A5495 occurs faster early in the disease course and reflects, in part, neuroaxonal loss and spinal cord dysfunction. ${ }^{3}$

The spinal cord is a clinically relevant site of the central nervous system and is often affected in multiple sclerosis. Focal and diffuse cord abnormalities, particularly in the cervical cord segment, have been described in up to $90 \%$ of patients with MS. ${ }^{4} \mathrm{MR}$ imaging measurement of cervical cord atrophy in patients with this disease provides valuable additional information related to disability that cannot be obtained from brain metrics. ${ }^{5}$ A progressive reduction of the cervical cord cross-sectional area (CSA) occurs in PPMS, ${ }^{6-9}$ and spinal cord atrophy has been shown to correlate with the severity of clinical disability. ${ }^{10-12}$ Moreover, some cross-sectional studies have reported that spinal cord atrophy is an independent predictor of disability progression. ${ }^{1,12}$

Nonetheless, the relationship between spinal cord area changes and worsening of disability has not been consistent among studies: Some authors describe an association, ${ }^{6,9}$ whereas others do 
not. ${ }^{7,8}$ This discrepancy may be explained by several factors, such as differing sample sizes, follow-up periods, and methods used to measure the cord CSA. ${ }^{7,13-15}$

Although some cross-sectional studies ${ }^{11,12}$ have demonstrated the value of spinal cord atrophy as an independent predictor of clinical outcome, only a few longitudinal studies ${ }^{9,16,17}$ have specifically focused on analyzing the clinical relevance of this finding in patients with MS, and these include a short follow-up or were not focused on PPMS.

Another factor to be taken into account is the method used for measuring the cervical cord CSA, because the optimal approach has not yet been identified. One of the most recently used is the proposed method of Horsfield et al, ${ }^{18}$ based on application of active surface models and known as the active surface method. With this approach, the CSA can be measured at specific levels and along extended portions of the cord with lower variability than other methods used in this region, such as the one proposed by Losseff et al. ${ }^{10}$ Some recent studies ${ }^{11,17,19}$ have used the active surface method for this purpose.

The aim of this study was to quantify the development of cervical cord atrophy and evaluate its association with the progression of clinical disability long term in patients with PPMS.

\section{MATERIALS AND METHODS}

\section{Subjects}

Thirty-one patients with PPMS were included in the study. These patients had been initially enrolled in a 2 -year, double-blind, placebo-controlled, Phase II pilot study, in which patients with PPMS or "transitional" forms of MS received either interferon $\beta-1 \mathrm{~b}$ at doses of $8 \mathrm{MIU}$ or a placebo for 24 months. ${ }^{20}$

This study was approved by the Vall d'Hebron Clinical Research Ethics Committee, who waived the requirement of written informed consent.

\section{Clinical Measures}

The Expanded Disability Status Scale (EDSS) score and disease duration were the clinical measures included. The EDSS was assessed at 3 time points: baseline and 2 years and approximately 14 years after baseline (14.12 \pm 2.88 years). Because EDSS values were not uniformly distributed across time, we used an averaged EDSS across time. To determine this value, we calculated the area under the curve of the EDSS. The area under the curve of the EDSS values was normalized to the maximum area under the curve of the EDSS in the time interval measured to obtain the normalized area under the curve of the EDSS (NAUCEDSS) value according to the following expression:

$$
\text { NAUCEDSS }=\frac{\sum_{k=0}^{1} \sum_{k}^{1}\left(\left[t_{\mathrm{k}+1}-t_{\mathrm{k}}\right]\left[E^{2} S S_{\mathrm{k}+1}+E D S S_{\mathrm{k}}\right]\right)}{10\left(t_{2}-t_{0}\right)},
$$

where $t_{\mathrm{k}}$ is the number of months from baseline $\left(t_{0}\right)$ in the time point $k=(0,1,2)$, and $\operatorname{EDSS}_{\mathrm{k}}$ is the EDSS measurement at the time point $k$.

Moreover, to obtain a prediction of long-term EDSS change, the differences between the last measurement and the baseline EDSS measurement were computed for each patient.

\section{MR Imaging Acquisition}

Two MRIs were analyzed in this group of patients, a baseline examination and a follow-up one obtained $6.30 \pm 0.23$ years (range, 5.92-7.17 years) after the baseline study.

All MR imaging studies were performed on a $1.5 \mathrm{~T}$ magnet (Magnetom Vision Plus; Siemens, Erlangen, Germany) using a quadrature transmit/receive head coil for the brain studies and a quadrature receive-only neck phased array coil for the cervical studies. In each brain examination, we obtained the following sequences: a transverse, T2-weighted, dual-echo turbo spin-echo sequence (TR, $3000 \mathrm{~ms}$; TE, 14-85 ms; echo-train length, 5; acquisitions, 1); and a transverse T1-weighted, spin-echo sequence (TR, $667 \mathrm{~ms}$; TE, $12 \mathrm{~ms}$; acquisitions, 2). For both sequences, 46 interleaved contiguous axial sections with $3-\mathrm{mm}$ thickness were acquired covering the whole brain, with a $192 \times 256$ matrix and 250-mm FOV, yielding an in-plane spatial resolution of approximately $1 \times 1 \mathrm{~mm}^{2}$. Following the brain study, a 3D volume image centered on the cervical spine was obtained using a magnetization-prepared rapid acquisition of gradient echo sequence with 128 partitions in the sagittal plane of $1.25-\mathrm{mm}$ thickness and the following parameters: TR/TE/TI, 9.7/450/4.2 ms; flip angle, 15; 256 phase-encodings in the $\mathrm{z}$-direction; 1 average; 250 -mm FOV; $256 \times 256$ matrix.

\section{MR Imaging Analysis}

Cross-sectional area measurements were assessed using a semiautomatic segmentation method based on an active surface model of the cord surface with intrinsic smoothness constraints, ${ }^{18}$ provided in the Jim 6.0 software package (http://www.xinapse.com/ home.php). Briefly, the sagittal 3D T1-weighted scans of the cervical cord from each patient were first reformatted in the axial plane and resampled to $1-\mathrm{mm}$ section thickness. Then, the active surface method was applied to each scan to estimate the cord surface and cord centerline (Fig 1). An initial estimate of the cord centerline was manually provided by placing landmarks at the extremes of the cord region to be studied and at approximately each $10 \mathrm{~mm}$ between these landmarks. Thus, the region studied comprised the segment from the most cranial section in which the odontoid process was visible down to the $\mathrm{C} 5$ superior margin. A single operator placed all landmarks. The cord centerline and cord outlines at each section were calculated using a segmentation algorithm, with steadily increasing refinement of the active surface model describing the cord outline. The total cord length was calculated in each region as the distance along the centerline between the upper and lower landmarks. In each region, the mean cervical cord CSA was calculated as the total cord volume divided by the cord length, and CSA was also measured at the $\mathrm{C} 2-\mathrm{C} 3$ (CSA23), C3-C4 (CSA34), and C4-C5 (CSA45) discs. CSA measurements were then normalized (NMCSA, NCSA23, NCSA34, NCSA45) - in a manner similar to the proposal of Lin et al ${ }^{21}$ - to the intracranial cross-sectional area measured at the inferior margins of the corpus callosum in an axial slice of the proton densityweighted image of each patient, as previously suggested. ${ }^{18}$ This adjustment was performed because cranial size was found to significantly correlate with the cord area in healthy controls. ${ }^{22}$ In addition to the normalized CSA measurements, the annualized (normalized) CSA loss rates between the baseline and follow-up 

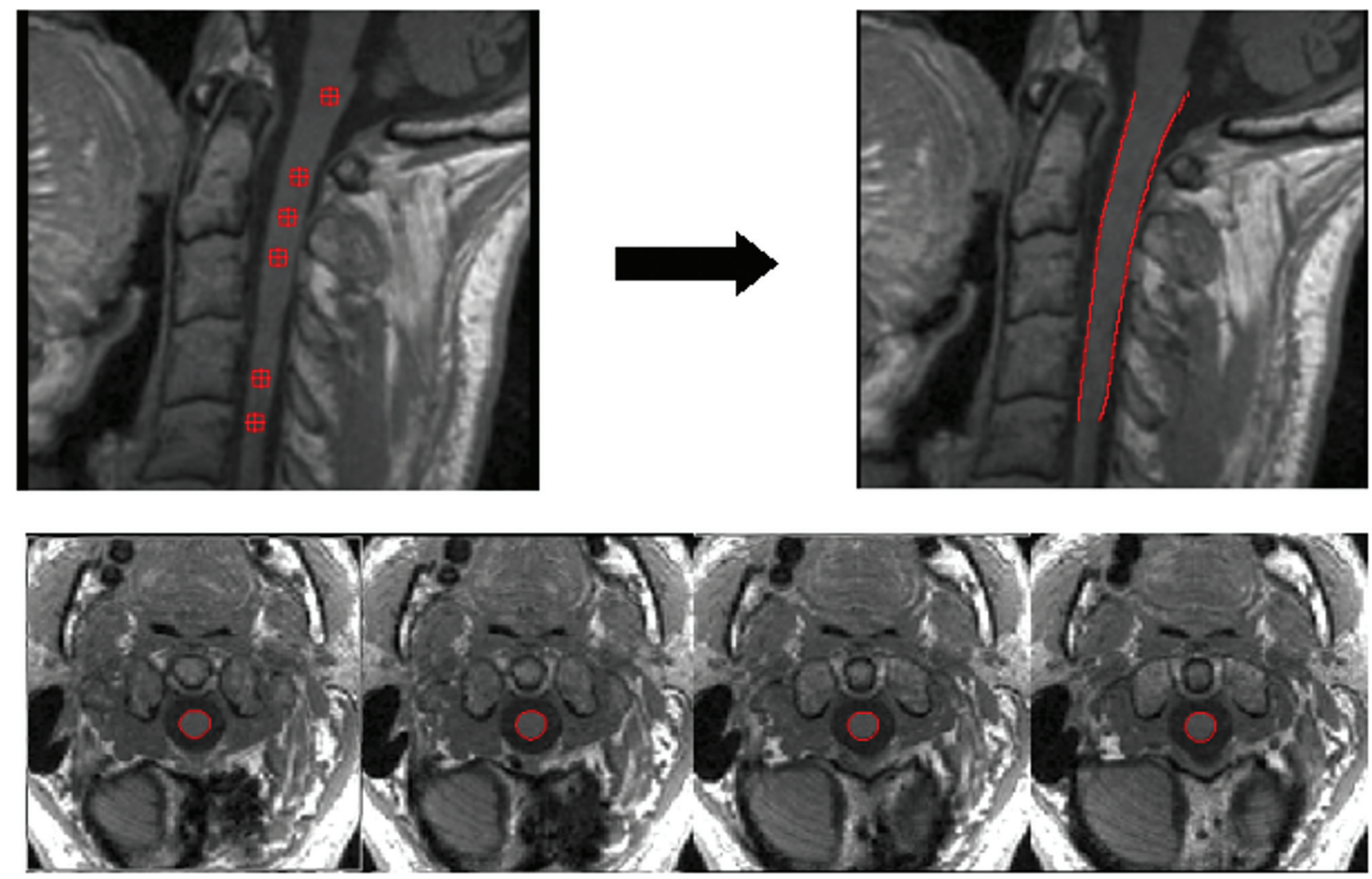

FIG 1. An example of the cord surface estimation obtained using the active surface method. The upper left image shows the location of the landmarks (red markers) that can be visualized in this sagittal slice. These landmarks were manually placed in the axial slices at the center area of the cord with a distance between them of approximately $10 \mathrm{~mm}$. The upper right image shows the cord outline estimation (red lines). The lower axial slices show some examples of the spinal cord segmentation obtained (region within red contour).

examination (aNMCSA, aNCSA23, aNCSA34, aNCSA45) were also evaluated.

To calculate the brain T2 lesion volume (T2LV), the same neuroradiologist initially analyzed and marked lesions found on brain MR imaging on the proton density-weighted hard copies with cross-reference to the T2-weighted images. All lesions marked on the hard copies were outlined on the computer image using a semiautomatic local thresholding contour technique (D.L. Plummer; University College, London, England) ${ }^{23}$; in cases in which the lesion could not be outlined satisfactorily with this approach, manual outlining was performed. A computer program then summed all the individual lesion volumes, and a final T2LV was generated and stored in a data base specifically designed for the study. To calculate the T1 lesion volume (T1LV), we used an automatic segmentation algorithm that measures T1LV from the initial T2 lesion segmentation that was used as a lesion mask. ${ }^{24}$

Brain atrophy was evaluated by measuring the brain parenchymal fraction (BPF) according to a previously described algorithm. $^{25}$

\section{Statistical Analysis}

To evaluate the longitudinal cord CSA changes, differences between the baseline and follow-up CSA measurements were assessed using a $t$ test for paired samples. Correlations of the cord CSA with the clinical and brain MR imaging measures were assessed using the Pearson correlation coefficient. To create a pre-
Table 1: Main baseline demographic, clinical, and conventional MRI characteristics

\begin{tabular}{lc}
\hline & Patients with MS \\
\hline Median age (range) (yr) & $51(33-61)$ \\
Men/women & $19: 12$ \\
Mean disease duration at baseline (range) $(\mathrm{yr})$ & $11.74(2-33)$ \\
Median EDSS score at baseline (range) & $5.5(3.0-6.5)$ \\
Mean brain T2LV at baseline (SD) (mL) & $18.12(20.63)$ \\
Mean brain TILV at baseline (SD) (mL) & $7.41(8.47)$ \\
Mean BPF at baseline (SD) & $73.13 \%(5.86 \%)$ \\
\hline
\end{tabular}

dictive model for the $\triangle$ EDSS, linear regression analysis was performed. A stepwise method was used to select the most relevant measures among the following: baseline CSA measures, annualized cord CSA loss rates, brain MR imaging measures, disease duration, and baseline EDSS scores. Age at baseline was also introduced in the model as a covariate. All statistical analyses were performed using SPSS (IBM, Armonk, New York). $P$ values $<.05$ were considered statistically significant.

\section{RESULTS}

\section{Clinical and Conventional MR Imaging Measures}

The main baseline demographic, clinical, and conventional MR imaging characteristics of the patients are shown in Table 1. During clinical follow-up, the median EDSS score was 6.0 (range, $4.0-8.5$ ) at 24 months and 7.5 (range, 4.0-9.5) at last measurement, approximately 14 years after baseline (14.12 \pm 2.88 years) (Table 2). The mean $\mathrm{T} 2$ and $\mathrm{T} 1$ lesion volumes increased to 
$21.56 \pm 20.65 \mathrm{~mL}$ and $9.53 \pm 9.32 \mathrm{~mL}$, respectively, whereas the mean BPF decreased by $3.83 \%$ at $6-7$ years of radiologic follow-up (Table 3). The T2LV, T1LV, and BPF changes were statistically significant $(P<.001)$.

\section{Table 2: Evolution of the EDSS}

\begin{tabular}{lccc}
\hline & Baseline & 2 Years & 14 Years \\
\hline $\begin{array}{l}\text { Median EDSS score } \\
\text { (range) }\end{array}$ & $5.5(3.0-6.5)$ & $6.0(4.0-8.5)$ & $7.5(4.0-9.5)$ \\
\hline
\end{tabular}

Table 3: MRI characteristics at follow-up

\begin{tabular}{lc}
\hline & Follow-Up \\
\hline Mean brain T2LV (SD) (mL) & $21.56(20.65)$ \\
Mean brain TILV (SD) (mL) & $9.53(9.32)$ \\
Mean BPF (SD) & $70.33 \%(5.84 \%)$ \\
\hline
\end{tabular}

Table 4: Longitudinal cross-sectional area measurements

\begin{tabular}{lcccc}
\hline & NMCSA & NCSA23 & NCSA34 & NCSA45 \\
\hline Mean at baseline (SD) $\left(\mathrm{mm}^{2}\right)$ & $71.49(6.37)$ & $67.76(7.26)$ & $68.21(7.39)$ & $71.51(8.27)$ \\
Mean at follow-up (SD) $\left(\mathrm{mm}^{2}\right)$ & $68.12(8.91)$ & $65.40(10.27)$ & $65.23(10.61)$ & $67.95(9.97)$ \\
$\begin{array}{l}\text { Cord area change averaged by } \\
\quad-0.77(1.14)\end{array}$ & $-0.62(1.20)$ & $-0.74(1.47)$ & $-0.77(1.61)$ \\
$\quad \begin{array}{l}\text { year (SD) (\%) } \\
P \text { value }\end{array}$ & .001 & .006 & .009 & .006 \\
\hline
\end{tabular}

\section{Longitudinal Changes in Cervical Cord Cross-Sectional \\ Area}

Baseline CSA measurements, follow-up CSA measurements, and cord area changes averaged by year are shown in Table 4 . The normalized CSA values decreased significantly in all the regions studied (Fig 2). The annualized CSA loss rates were similar for all the normalized CSA measurements.

\section{Associations between Measurements}

According to the Evans categorization system, ${ }^{26}$ the normalized CSA measurements showed a weak-to-moderate negative association with the normalized area under the curve of the EDSS at baseline (NMCSA: $r=-0.357, P=.049$; NCSA23: $r=-0.418$, $P=.019)$ and at follow-up (NMCSA: $r=-0.439, P=.013$; NCSA23: $r=-0.408, P=.023$; NCSA34: $r=-0.387, P=.032$ ).

No significant correlations were found between the annualized (normalized) cord CSA loss rates and the normalized area under the curve of the EDSS. Analysis of associations between the normalized CSA and brain MR imaging measurements showed no associations of the
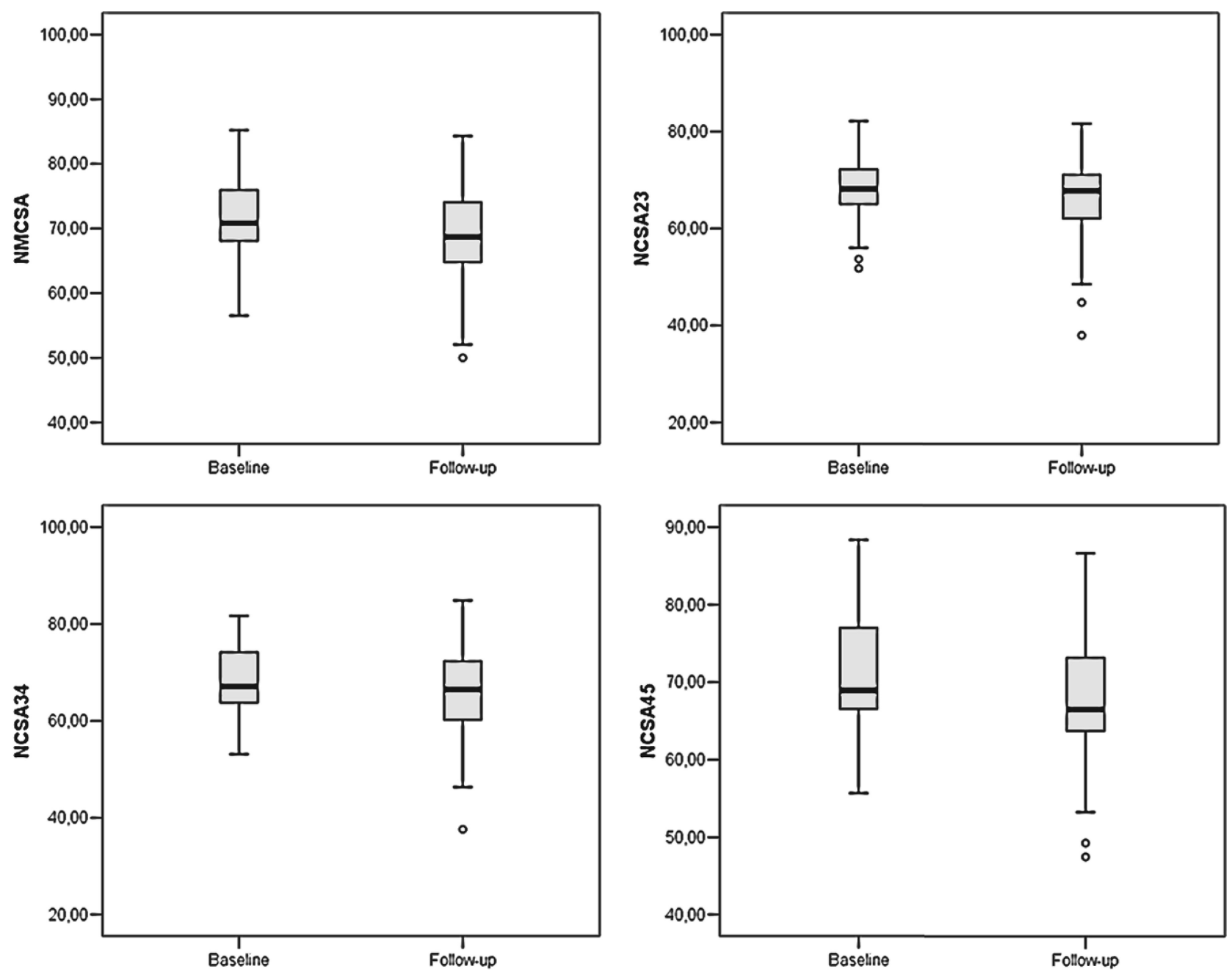

FIG 2. Box-and-whisker plots of normalized cross-sectional areas: NMCSA (upper left), NCSA23 (upper right), NCSA34 (lower left), and NCSA45 (lower right). 
BPF, T2LV, or T1LV with the various CSA measurements. A moderate correlation was found between the baseline BPF and the annualized (normalized) CSA loss rates at C2-C3 $(r=-0.419$, $P=.019)$ and C3-C4 $(r=-0.425, P=.017)$, whereas a trend was observed between the baseline BPF and the aNMCSA $(r=$ $-0.355, P=.05)$.

\section{Prediction of Long-Term EDSS Change}

The long-term EDSS change was measured $14.12 \pm 2.88$ years after baseline to obtain an estimation on a time horizon of around 14 years. In the linear regression analysis to predict this change, the annualized mean cervical cord area loss rate ( $a N M C S A)$ and baseline EDSS $\left(E D S S_{0}\right)$ were introduced in the model using the stepwise method, and age was introduced as a covariate. However, only aNMCSA and EDSS ${ }_{0}$ with $P$ values of .007 and .025 , respectively, were statistically significant variables. The model obtained was then defined by the following expression:

$\Delta E D S S=4.0-0.628$ aNMCSA

$$
\text { - 0.476 } \mathrm{EDSS}_{0}+0.006 \text { Age. }
$$

The F-test showed a significant $P$ value (.004); thus, it was assumed that there was a linear relationship among the variables in our model. Finally, $R^{2}=0.390$, which indicated that the linear regression model explained $39.0 \%$ of the total variance in the data.

\section{DISCUSSION}

The present study investigated the role of cervical cord atrophy in a longitudinal study of a cohort of patients with PPMS to evaluate the association of this MR imaging finding with clinical progression of disability and with other MR imaging measures. We found a significant decrease in the normalized CSA values in all the regions studied. Moreover, some weak-to-moderate correlations were found between the weighted-average EDSS and normalized CSA values both at baseline and follow-up.

The annual loss of spinal cord tissue in our cohort was lower than that reported in previous studies. ${ }^{6,8,9,13,17,21,22}$ However, Lukas et $\mathrm{al}^{9}$ recently reported that spinal cord tissue loss may slow down with time, with already highly atrophied structures exhibiting slower atrophy rates. Our results support this notion: During the lengthy follow-up period, we found a smaller annualized CSA loss than other studies with a shorter follow-up.

To obtain the weighted-average EDSS, we used the area under the curve. The area under the curve becomes interesting when the longitudinal time intervals are unequally spaced, as was the case in the present study. Then the area under the curve reflects the weighted average of a certain outcome variable during the total follow-up period. ${ }^{27}$ Area under the curve values were also normalized to facilitate their interpretation by an index in a range from 0 to 1 , associated with patient disability.

Although the linear regression model predicting long-term EDSS change showed a strong correlation with the data according to the Evans categorization, ${ }^{26}$ the only significant variables remaining in the model were the aNMCSA and baseline EDSS. None of the CSA measures or conventional MR imaging measures showed high enough significance to be included in the model. The model showed that the greater the progression of cervical cord atrophy, associated with a more negative aNMCSA, the greater was the long-term EDSS change. Baseline EDSS acted as a limiting factor in the model, because the margin to a long-term EDSS increase was lower when baseline EDSS values were higher.

The lack of association between the BPF and cross-sectional area values is in agreement with a previous study in patients with PPMS. $^{28}$ At C2-C3 and C3-C4, a moderate negative correlation between the BPF and the annualized rate of spinal cord tissue loss was observed, whereas only a trend was seen between the baseline BPF and aNMCSA. These findings may be an indication that brain and cord pathology evolve differently and that measurement of brain and cord atrophy can provide complementary information about the severity of PPMS. ${ }^{28}$

The data analysis did not include the effect of treatment. This factor was not considered essential because the study patients were part of a cohort participating in a clinical trial ${ }^{20}$ in which one group was treated with interferon $\beta 1-\mathrm{b}$, and the remainder, with placebo during the first 2 years; and there were no differences in EDSS progression or CSA measurements between the groups.

This study had limitations. First, the long follow-up made it difficult to enroll a larger sample that fulfilled the clinical and radiologic information required for the study. Second, images were acquired using a $1.5 \mathrm{~T} \mathrm{MR}$ imaging scanner. Most recent studies use 3T scanners to acquire cervical cord images because they provide a better signal-to-noise ratio in the same acquisition time. However, given the duration of our study, a 1.5T scanner was used at the first time point and we decided to maintain the same scanner throughout the study. Third, the sequence used to acquire cervical cord images was 3D T1-weighted MPRAGE. In a recent study, Kearney et $\mathrm{al}^{19}$ proposed the use of phase-sensitive inversion recovery sequences to take advantage of their better resolution and higher contrast. It is likely that reproducibility would improve if the active surface method were combined with a phase-sensitive inversion recovery rather than a 3D T1-weighted MPRAGE sequence. However, because of the retrospective nature of the study, it was not possible to introduce this sequence in the acquisition. Nonetheless, Kearney et al mentioned that when phase-sensitive inversion recovery cannot be used, combining active surface with 3D MPRAGE may be the most suitable approach. Fourth, EDSS values were not uniformly distributed across time. We tried to resolve this problem using a weighted-average measurement (normalized area under the curve), but a uniform distribution would have allowed further analysis. Finally, inclusion of a healthy control group would have helped to differentiate decreases in cervical cord values due to age and decreases due to MS-induced atrophy.

\section{CONCLUSIONS}

The results of this study suggest that cervical cord area measures are associated with disability in patients with PPMS. More interesting, this study supports MR imaging changes being taken into account when developing predictive studies. Specifically, we found that the rate of cervical cord area loss could play a relevant role in predicting clinical disability progression long term. Further longitudinal studies focused on the evaluation of cervical cord area changes in patients with PPMS, and their relation to disease progression for long periods would help to define the 
value of cervical cord atrophy as a surrogate marker in MS clinical trials or for clinical management of these patients.

\section{ACKNOWLEDGMENTS}

The authors thank Celine Cavallo for English language support.

Disclosures: Cristina Auger-UNRELATED: Payment for Lectures Including Service on Speakers Bureaus: Novartis, Teva Pharmaceuticals, Biogen Idec, Stendhal. Jaume Sastre-Garriga_UNRELATED: Consultancy: Almirall; Grants/Grants Pending: Bayer AG, Sanofi Genzyme*; Payment for Lectures Including Service on Speakers Bureaus: Teva Pharmaceuticals, Biogen Idec, Novartis, Roche, Almirall, Merck Serono, Celgene, Sanofi Genzyme; Payment for Development of Educational Presentations: Merck Serono. Mar Tintoré-UNRELATED: Board Membership: Almirall, Bayer AG, Biogen Idec, Sanofi Genzyme, Merck Serono, Novartis, Sanofi-Aventis, Roche, Teva Pharmaceuticals; Grants/Grants Pending: Almirall, Bayer AG, Biogen Idec, Sanofi Genzyme, Merck Serono, Novartis, Sanofi Aventis, Roche, and Teva Pharmaceuticals*; Payment for Lectures Including Service on Speakers Bureaus: Almirall, Bayer AG, Biogen Idec, Sanofi Genzyme, Merck Serono, Novartis, Sanofi Aventis, Roche, and Teva Pharmaceuticals; Payment for Development of Educational Presentations: Almirall, Bayer AG, Biogen Idec, Sanofi Genzyme, Merck Serono, Novartis, Sanofi Aventis, Roche, and Teva Pharmaceuticals. Xavier Montalban-UNRELATED: Consultancy: Actelion, Almirall, Bayer AG, Biogen, Celgene, Hoffmann-La Roche, Merck Serono, Novartis, Oryzon Genomics, Sanofi Genzyme, Teva Pharmaceuticals. Alex Rovira-UNRELATED: Board Membership: Novartis, Sanofi Genzyme; Payment for Lectures Including Service on Speakers Bureaus: Bayer AG, Stendhal Americas, Sanofi Genzyme, Bracco, Merck Serono, Teva Pharmaceuticals, Novartis, Roche, Biogen Idec; Payment for Development of Educational Presentations: Biogen Idec. *Money paid to the institution.

\section{REFERENCES}

1. Thompson AJ, Polman $\mathrm{CH}$, Miller $\mathrm{DH}$, et al. Primary progressive multiple sclerosis. Brain 1997;120:1085-96 CrossRef Medline

2. Thompson AJ, Montalban X, Barkhof F, et al. Diagnostic criteria for primary progressive multiple sclerosis: a position paper. Ann Neurol 2000;47:831-35 Medline

3. Bjartmar C, Kidd G, Mörk S, et al. Neurological disability correlates with spinal cord axonal loss and reduced $\mathrm{N}$-acetyl aspartate in chronic multiple sclerosis patients. Ann Neurol 2000;48:893-901 Medline

4. Nijeholt GJ, van Walderveen MA, Castelijns JA, et al. Brain and spinal cord abnormalities in multiple sclerosis: correlation between MRI parameters, clinical subtypes and symptoms. Brain 1998;121: 687-97 CrossRef Medline

5. Zivadinov R, Banas AC, Yella V, et al. Comparison of three different methods for measurement of cervical cord atrophy in multiple sclerosis. AJNR Am J Neuroradiol 2008;29:319-25 CrossRef Medline

6. Ingle GT, Stevenson VL, Miller DH, et al. Primary progressive multiple sclerosis: a 5-year clinical and MR study. Brain 2003;126: 2528-36 CrossRef Medline

7. Stevenson VL, Ingle GT, Miller DH, et al. Magnetic resonance imaging predictors of disability in primary progressive multiple sclerosis: a 5-year study. Mult Scler 2004;10:398-401 CrossRef Medline

8. Agosta F, Absinta M, Sormani MP, et al. In vivo assessment of cervical cord damage in MS patients: a longitudinal diffusion tensor MRI study. Brain 2007;130:2211-19 CrossRef Medline

9. Lukas C, Knol DL, Sombekke MH, et al. Cervical spinal cord volume loss is related to clinical disability progression in multiple sclerosis. J Neurol Neurosurg Psychiatry 2015;86:410-18 CrossRef Medline

10. Losseff NA, Webb SL, O'Riordan JI, et al. Spinal cord atrophy and disability in multiple sclerosis: a new reproducible and sensitive
MRI method with potential to monitor disease progression. Brain 1996;119:701-08 CrossRef Medline

11. Rocca MA, Horsfield MA, Sala S, et al. A multicenter assessment of cervical cord atrophy among MS clinical phenotypes. Neurology 2011;76:2096-102 CrossRef Medline

12. Lukas C, Sombekke MH, Bellenberg B, et al. Relevance of spinal cord abnormalities to clinical disability in multiple sclerosis: MR imaging findings in a large cohort of patients. Radiology 2013;269:542-52 CrossRef Medline

13. Furby J, Hayton T, Altmann D, et al. A longitudinal study of MRIdetected atrophy in secondary progressive multiple sclerosis. $\mathrm{J} \mathrm{Neu}$ rol 2010;257:1508-16 CrossRef Medline

14. Sastre-Garriga J, Ingle GT, Rovaris M, et al. Long-term clinical outcome of primary progressive MS: predictive value of clinical and MRI data. Neurology 2005;65:633-35 CrossRef Medline

15. Stevenson VL, Miller DH, Leary SM, et al. One year follow up study of primary and transitional progressive multiple sclerosis. J Neurol Neurosurg Psychiatry 2000;68:713-18 CrossRef Medline

16. Cawley N, Tur C, Prados F, et al. Spinal cord atrophy as a primary outcome measure in Phase II trials of progressive multiple sclerosis. Mult Scler 2017 May 1. [Epub ahead of print] CrossRef Medline

17. Valsasina P, Rocca MA, Horsfield MA, et al. A longitudinal MRI study of cervical cord atrophy in multiple sclerosis. J Neurol 2015; 262:1622-28 CrossRef Medline

18. Horsfield MA, Sala S, Neema M, et al. Rapid semi-automatic segmentation of the spinal cord from magnetic resonance images: application in multiple sclerosis. Neuroimage 2010;50:446-55 CrossRef Medline

19. Kearney H, Yiannakas MC, Abdel-Aziz K, et al. Improved MRI quantification of spinal cord atrophy in multiple sclerosis. J Magn Reson Imaging 2014;39:617-23 CrossRef Medline

20. Montalban X, Sastre-Garriga J, Tintoré M, et al. A single-center, randomized, double-blind, placebo-controlled study of interferon beta- $1 \mathrm{~b}$ on primary progressive and transitional multiple sclerosis. Mult Scler 2009;15:1195-205 CrossRef Medline

21. Lin X, Tench CR, Turner B, et al. Spinal cord atrophy and disability in multiple sclerosis over four years: application of a reproducible automated technique in monitoring disease progression in a cohort of the interferon beta-1a (Rebif) treatment trial. J Neurol Neurosurg Psychiatry 2003;74:1090-94 CrossRef Medline

22. Rashid W, Davies GR, Chard DT, et al. Increasing cord atrophy in early relapsing-remitting multiple sclerosis: a 3 year study. J Neurol Neurosurg Psychiatry 2006;77:51-55 CrossRef Medline

23. Plummer DL. Dispimage: a display and analysis tool for medical images. Riv Neuroradiol 1992;19:1715-20

24. Aymerich FX, Sobrevilla P, Montseny E, et al. Fuzzy approach to lesion load measurement in T1-weighted images. Mult Scler 2001; 7(suppl 1):87

25. Aymerich X, Sobrevilla P, Montseny E, et al. Application of an automatic segmentation algorithm to brain atrophy assessment in multiple sclerosis. Rev Neurol (Paris) 2000;156(suppl 3):103-04

26. Evans JD. Straightforward Statistics for Behavioral Sciences. Pacific Grove: Brooks/Cole Publishing; 1996

27. Twisk JW. Applied Longitudinal Data Analysis for Epidemiology: A Practical Guide. New York: Cambridge University Press; 2013

28. Rovaris M, Judica E, Sastre-Garriga J, et al. Large-scale, multicentre, quantitative MRI study of brain and cord damage in primary progressive multiple sclerosis. Mult Scler 2008;14:455-64 CrossRef Medline 